\title{
La calidad del servicio bibliotecario y el desempeño académico en la universidad: un modelo explicativo
}

\author{
Eloisa Aparicio-Ley, Judith Cavazos-Arroyo y Martha-Leticia Gaeta-González
}

\section{RESUMEN}

El objetivo fue analizar si la calidad del servicio bibliotecario afecta el rendimiento académico de los estudiantes a través de cuatro constructos. Se desarrolló una investigación cuantitativa, explicativa, transversal y un muestreo por conveniencia a 701 estudiantes universitarios mexicanos. Se analizaron los datos con un modelo de ecuaciones estructurales. Se encontró efecto significativo de la calidad del servicio bibliotecario en las creencias de autoeficacia de los estudiantes, lo que impacta en su participación en la biblioteca y en sus estrategias de aprendizaje. Esto incide en los procesos de autorregulación del aprendizaje, lo que a su vez afecta el rendimiento académico. Se muestran efectos directos e indirectos de la calidad del servicio bibliotecario en aspectos del proceso académico, impactando finalmente en el rendimiento académico.

Palabras clave: rendimiento académico, calidad, biblioteca universitaria, autoeficacia, participación del estudiante, estrategias de aprendizaje, aprendizaje autorregulado, México.

\section{Eloisa Aparicio-Ley}

Mexicana. Maestra en Dirección y Mercadotecnia, Universidad Popular Autónoma del Estado de Puebla, México. Docente, Universidad Popular Autónoma del Estado de Puebla, México. Temas de investigación: calidad en el servicio, emprendimiento social. ORCID: https://orcid.org/0000-0003-4992-3458.

\section{Judith Cavazos-Arroyo}




\title{
A qualidade do serviço bibliotecário e o desempenho académico na universidade: um modelo explicativo
}

\section{RESUMO}

O objetivo foi analisar se a qualidade do serviço bibliotecário afeta o rendimento acadêmico dos estudantes através de quatro constructos. Desenvolveu-se uma pesquisa quantitativa, explicativa, transversal e uma amostragem por conveniência a 701 estudantes universitários mexicanos. Analisaram-se os dados com um modelo de equações estruturais. Encontrou-se efeito significativo da qualidade do serviço bibliotecário nas crenças de autoeficácia dos estudantes, o que impacta em sua participação na biblioteca e em suas estratégias de aprendizagem. Isto incide nos processos de autorregulação da aprendizagem, o que por sua vez afeta o rendimento acadêmico. Mostram-se efeitos diretos e indiretos da qualidade do serviço bibliotecário em aspectos do processo acadêmico, impactando finalmente no rendimento acadêmico.

Palavras chave: rendimento acadêmico, qualidade, biblioteca universitária, autoeficácia, participação do estudante, estratégias de aprendizagem, aprendizagem autorregulada, México.

\section{Library service quality and academic performance at university: an explanatory model}

\begin{abstract}
The purpose of this article was to analyze in which way the quality of library service affects students' academic performance through four constructs. A quantitative, explanatory, cross-sectional, convenience, and explanatory sample of 701 Mexican university students was carried out and data were analyzed with structural equation modeling. A significant effect of library service quality on students' self-efficacy beliefs was found, which impacts their participation in the library and their learning strategies. This also has an impact on self-regulatory learning processes, which in turn affect academic performance. The results show the direct and indirect effects of the quality of library services on aspects of the academic process, which ultimately impacts academic performance.
\end{abstract}

Key words: academic performance, quality, university library, self-efficacy, student participation, learning strategies, self-regulated learning, Mexico. 


\section{Introducción}

El rendimiento académico ha sido uno de los temas más estudiados en el campo educativo (Vargas y Montero, 2016). Las investigaciones se han centrado en conocer sus antecedentes; ya que se sabe que muchos constructos le afectan directa e indirectamente. Investigaciones previas (Duggal y Mehta, 2015) muestran que las instalaciones universitarias, como el laboratorio de computación y la biblioteca física, son uno de los factores que impactan en el rendimiento académico de los estudiantes.

Las instalaciones cobran importancia principalmente en dos momentos, cuando la institución las proporciona a los estudiantes y la segunda, cuando los estudiantes utilizan esas instalaciones (Duggal y Mehta, 2015). Por lo que conocer la percepción de los estudiantes sobre la calidad de los servicios que les proporciona la biblioteca académica y su incidencia en los procesos académicos, vinculada al uso que dan a los mismos, pueden proporcionar información relevante sobre su rendimiento académico en la universidad.

Actualmente, debido a la inserción de tecnologías de la información, la biblioteca física presenta nuevos desafíos en cuanto a su objetivo principal, ya que se ha convertido en un espacio social y multifuncional donde los estudiantes se reúnen para realizar diversas tareas (Varela y Baiget, 2012). Así, en los últimos años el servicio bibliotecario se ha modificado, creándose bibliotecas digitales para satisfacer las necesidades académicas (González y Molina, 2008).

En este punto, es importante resaltar la importancia de este espacio en la vida académica como apoyo en las actividades de aprendizaje e investigación de estudiantes, docentes e investigadores a través de una variedad de recursos y servicios. Además, como se mencionó anteriormente, se ha reconocido que el uso de la biblioteca académica está relacionado con el rendimiento académico de los estudiantes (Association of College \& Research Libraries (ACRL),
2016). Sin embargo, a pesar de la gran cantidad de investigaciones sobre este tema en el campo académico, no hay publicaciones que muestren cómo las instalaciones bibliotecarias y los servicios que ofrece, apoyan las labores de aprendizaje y el rendimiento académico, a través de la participación de los estudiantes en este servicio.

Con base en los argumentos anteriores, el objetivo de esta investigación consiste en analizar si la calidad del servicio de la biblioteca afecta el rendimiento académico de los estudiantes, a través de su incidencia en la autoeficacia del alumnado, participación en la biblioteca, estrategias de aprendizaje y proceso de autorregulación del aprendizaje.

\section{Calidad del servicio bibliotecario y autoeficacia}

La biblioteca académica es un servicio complementario en las universidades que facilita las labores para apoyar los procesos de aprendizaje y enseñanza, a través de material digital, impreso y herramientas tecnológicas (American Library Association (ALA), 2010). La biblioteca continuamente se enfrenta al reto de demostrar a la comunidad la calidad de sus servicios, la cual se ha definido de acuerdo con la satisfacción y expectativas de sus usuarios. En este sentido se realizan esfuerzos para identificar más claramente la influencia de la calidad de los servicios bibliotecarios en los resultados institucionalmente relevantes: la productividad del profesorado o los resultados académicos de los estudiantes.

Para evaluar la calidad de la biblioteca (en términos de satisfacción y expectativas) se ha creado la escala LibQUAL +; a través de ella, los usuarios califican tres dimensiones de la biblioteca: afectiva, espacial y control de la información (LibQUAL+, 2016). LibQUAL + ha sido utilizada por más de 1200 instituciones en todo el mundo, lo que les ha permitido mejorar el servicio y brindar una mayor respuesta a las necesidades de sus usuarios (Green y Kyrillidou, 2012). 
Por otro lado, en cuanto a los resultados académicos de los estudiantes, como nos recuerda Bandura (1993), las creencias de los estudiantes sobre sí mismos y la autoconfianza que desplieguen sobre su capacidad intelectual se consideran factores fundamentales para el logro académico. Así, más allá de las capacidades que la persona posee, los juicios sobre lo que se considera capaz de lograr a partir de ellas (su autoeficacia), constituyen un aspecto fundamental para la consecución y logro de una actividad académica en particular.

Las creencias de autoeficacia se definen como el conjunto de expectativas y juicios que una persona crea sobre sus propias capacidades para realizar acciones determinadas con éxito (Blanco, 2010), lo cual tiene efectos significativos sobre las motivaciones, las emociones y los comportamientos personales (HyangIl y Kyung-Ae, 2017) y a su vez podría estar afectada por factores personales, sociales o ambientales.

La autoeficacia tiene implicaciones en varios aspectos de la vida personal y académica del alumnado, como la amistad, la consecución académica, las elecciones vocacionales y el rendimiento escolar (Pajares y Urdan, 2006). En el campo académico, los estudiantes con alto grado de autoeficacia perseveran en el logro de objetivos, a pesar de los obstáculos; por lo tanto, este constructo ha mostrado tener un efecto directo e indirecto sobre el rendimiento académico (Hyang-Il y Kyung-Ae, 2017). Además, se ha demostrado que la autoeficacia incide en cómo se procesa la información (por ejemplo, el grado de elaboración y profundización), así como en las estrategias de aprendizaje que se utilizan (Akilli y Genç, 2017; González, 1997) y es un predictor de la autorregulación (Zimmerman y Martinez-Pons, 1990) y de la co-creación (Alves, Ferreira y Fernandes, 2016).

La evidencia científica ha demostrado también que las experiencias sociales preceden a la autoeficacia y determinan si alguien tiene niveles altos o bajos de ella (Zulkosky, 2009). Así, una fuerte autoeficacia puede estar influida por ciertas percepciones positivas (Luszczynska, Scholz y Schwarzer, 2005), como las experiencias provenientes de la interacción sustentada en la calidad del servicio. Tal es el caso del servicio brindado por la biblioteca universitaria, ya que los estudiantes pueden llegar a sentirse más confiados y seguros de su capacidad académica si perciben que los atributos tangibles e intangibles ofrecidos por ésta facilitan y apoyan sus procesos de estudio y aprendizaje (Zha et al., 2015). Investigaciones empíricas han demostrado que la autoeficacia se ve afectada por una experiencia positiva respecto a la calidad y el servicio de información (Young Kim y Seok Lee, 2013; Zha et al., 2015), lo que está estrechamente relacionado con el servicio de la biblioteca. Por ello, es posible suponer que:

- H1: La percepción de la calidad del servicio de la biblioteca es un antecedente de las creencias de autoeficacia.

\section{Participación del estudiante en la biblioteca}

La participación de los estudiantes en la biblioteca puede entenderse como el grado en que ellos se involucran en el servicio de la biblioteca y son co-creadores en ese proceso (Encinas-Orozco y Cavazos-Arroyo, 2017). Por lo tanto, a través de la participación, los usuarios pueden co-crear valor dentro de la biblioteca, como proporcionar información esencial para que los empleados puedan ayudar mejor a los estudiantes (Alves et al., 2016).

La participación de los alumnos en la biblioteca contribuye a su involucramiento activo en el proceso educativo, por lo que podría ayudarles a estar más motivados, mejorar sus procesos de aprendizaje y obtener mejores calificaciones (Rocca, 2010). Estudios en otras disciplinas han demostrado que la co-creación dentro de la organización se ve afectada por la autoeficacia (Alves et al., 2016), también se ha comprobado que la autoeficacia tiene un efecto en la participación de los estudiantes por medio del 
aprendizaje en línea (Kui y Kun, 2014) y de igual manera se verificó el mismo efecto en un estudio sobre la participación ciudadana (Yeung, Passmore y Packer, 2012). Por lo tanto, de acuerdo con los planteamientos anteriores, se propone la siguiente hipótesis:

- H2a: Las creencias de autoeficacia tienen un efecto positivo en la participación de los estudiantes en la biblioteca.

\section{Estrategias de aprendizaje}

Las estrategias de aprendizaje son actividades y métodos que ayudan a las personas a aprender por sí mismas (Akilli y Genç, 2017), los estudiantes las utilizan para seleccionar, organizar e integrar datos con el fin de construir conocimiento basado en la información comprendida (ibid.). No se refiere a procesos, como la comprensión o atención, ni a técnicas como hacer un resumen, sino a actividades mentales y cognitivas que requieren un plan de acción (Roux y Anzures, 2015).

Existen estrategias para adquirir, codificar y recuperar información, así como estrategias para apoyar el procesamiento de la información. Además, las estrategias de aprendizaje están directamente relacionadas con el proceso de enseñanza y aprendizaje, por lo que afectan la calidad del aprendizaje y el desarrollo académico (Visbal-Cadavid, MendozaMendoza y Díaz-Santana, 2017).

De acuerdo con investigaciones previas, las estrategias de aprendizaje son afectadas directamente por la autoeficacia (Akilli y Genç, 2017); se ha demostrado que ésta es un predictor de las estrategias de procesamiento de la información, lo que a su vez tiene un efecto significativo sobre la autorregulación (Mellat y Lavasani, 2011). De hecho, el conocer diferentes estrategias de aprendizaje e identificar cuáles son las más apropiadas para diferentes situaciones constituye una parte importante del aprendizaje autorregulado (García, McCann, Turner y Roska, 1998). Por lo tanto, se presenta la siguiente hipótesis:
- H2b: Las creencias de autoeficacia tienen una incidencia en las estrategias de aprendizaje.

\section{Procesos de autorregulación del aprendizaje}

La autorregulación del aprendizaje es un proceso en el que los estudiantes autogeneran y toman control de sus pensamientos, sentimientos y acciones para optimizar y lograr sus objetivos de aprendizaje. Durante este proceso, los estudiantes también controlan y monitorean la efectividad de sus estrategias de aprendizaje (Zimmerman y Schunk, 2008) por medio de tres fases (Cassidy, 2011): previsión, desempeño y autorreflexión. En la fase de previsión, los estudiantes planean, establecen objetivos, seleccionan técnicas de aprendizaje y encuentran motivación para realizar la tarea. Durante el desempeño, los estudiantes verifican si las estrategias elegidas fueron apropiadas para lograr sus objetivos y, finalmente, la autorreflexión ocurre cuando los estudiantes, de acuerdo con su percepción de los resultados, identifican las mejores técnicas de aprendizaje, proceso importante en la motivación y selección de estrategias apropiadas para futuras tareas (Gandomkar et al., 2016).

Se ha evidenciado que la participación activa de los estudiantes afecta la autorregulación en la educación a distancia (Chih-Yuan Sun y Rueda, 2012) y que la co-creación de los estudiantes beneficia su autorregulación (Fraile, Panadero y Pardo, 2017). Además, se ha demostrado que el procesamiento estratégico tiene un efecto sobre la autorregulación (Mellat y Lavasani, 2011). Con base en lo anterior, las hipótesis planteadas son:

- H3a: La participación de los estudiantes en la biblioteca es un predictor de los procesos de autorregulación del aprendizaje.

- H3b: Las estrategias de aprendizaje afectan directamente los procesos de autorregulación del aprendizaje. 


\section{Rendimiento académico}

El rendimiento académico ha sido un tema relevante debido a su influencia en la formación del capital humano y en la sociedad en general, además contribuye en el aumento de la productividad y la igualdad social (Hojo, 2012). Este constructo se define como el nivel de rendimiento o la puntuación obtenida al final de un estudio determinado en un cierto periodo de tiempo, por ejemplo, un semestre o un programa académico (Fiagborlo y Kunu, 2016).

El estudio del rendimiento académico es difícil debido a los diversos factores que lo afectan, como son las características de los estudiantes, la calidad del programa escolar, la formación de los docentes y los antecedentes familiares de los estudiantes, entre otros (Zimmer y Haumann, 2013). La medida más común del rendimiento académico de los estudiantes es el promedio de calificaciones (GPA) (Fiagborlo y Kunu, 2016). Algunos investigadores han demostrado la relación directa entre la autorregulación y el rendimiento académico (Duru, Duru y Balkis, 2014), también se ha demostrado que los estudiantes con una mayor autorregulación presentan mayores logros académicos (Montroy, Bowles, Skibbe y Foster, 2014). Por lo tanto, se propone la siguiente hipótesis:

- H4: El proceso de autorregulación del aprendizaje es un antecedente del rendimiento académico.

\section{Metodología}

Para responder al objetivo de esta investigación, que consiste en analizar si la calidad del servicio de la biblioteca afecta el rendimiento académico de los estudiantes, a través de su incidencia en la autoeficacia del alumnado, participación en la biblioteca, estrategias de aprendizaje y proceso de autorregulación del aprendizaje, se desarrolló una investigación cuantitativa, explicativa y transversal mediante un modelo de ecuaciones estructurales (Hair, Bush y Ortinau,
2010). La investigación se llevó a cabo en cuatro universidades privadas en México. La muestra se seleccionó a través de un muestreo no probabilístico por conveniencia, de acuerdo con la población estudiantil de licenciatura de cada universidad (Malhotra, 2008). La recolección de datos se realizó a través de una encuesta personal durante el otoño de 2016 y la primavera de 2017. El cuestionario fue diseñado con base en diferentes escalas (tabla 1).

A partir de una población de 16300 estudiantes, un error de precisión del 4\% y un nivel de confianza del 97\%, se obtuvo una muestra de 701 estudiantes. Para el análisis de datos, se empleó el método de mínimos cuadrados parciales, utilizando el paquete estadístico SmartPLS 3.0.

\section{Análisis de resultados Resultados demográficos}

La mayoría de los estudiantes encuestados estudian licenciaturas del decanato de ingeniería, ciencias de la salud o economía. Una minoría de ellos estudia en los decanatos de ciencias biológicas, artes y humanidades y ciencias sociales. El 52.5\% de los participantes cursaba los primeros cuatro semestres de su carrera, el resto se encontraba entre el quinto y onceavo semestre. El $65.8 \%$ de la muestra tenía entre 20 y 24 años, el 32.2\% tenía entre 15 y 19 años y sólo dos estudiantes tenían más de 25 años. De la muestra total, el $54.3 \%$ son mujeres y el $45.7 \%$ son hombres.

\section{Resultados del modelo de medida}

Los resultados presentados a continuación corresponden a los 58 ítems elegidos entre los 86 ítems iniciales, que tienen una carga $(\lambda)$ mayor a 0.70 , lo cual denota una buena fiabilidad individual. Igualmente se analizaron el alfa de Cronbach (a) y la fiabilidad compuesta ( $\rho c)$. Ambas medidas indican buena consistencia interna, con valores de alfa de Cronbach entre 0.70 y 0.90 (Celina y Campo, 2005) y valores de fiabilidad compuesta que exceden el umbral establecido de 0.70 (Nunnally, 1978), como se muestra 
en la tabla 2. Además, se evaluó la validez convergente y discriminante de los constructos. La validez convergente se midió por medio de la varianza extraída media (AVE). Los resultados que se muestran en la tabla 2, muestran valores que superan el umbral establecido de 0.50 (Fornell y Larcker, 1981).

Para analizar la validez discriminante, se llevaron a cabo tres pruebas. La primera fue el criterio de Fornell-Larcker; que afirma que las correlaciones entre cada par de constructos deben ser menores a la raíz cuadrada del AVE de cada constructo (Fornell y Larcker, 1981). Los resultados de esta prueba se muestran en la tabla 3. Hay cinco valores resaltados que no satisfacen el criterio, pero corresponden a los constructos de segundo orden; por ejemplo, la calidad del servicio de la biblioteca (B) con sus dimensiones afectiva (Ba) y espacial (Be). También creencias de autoeficacia $(\mathrm{C})$ con su dimensión de autoeficacia general $(\mathrm{Cg})$ y participación del alumno en la biblioteca $(\mathrm{P})$, con su dimensión de comportamiento responsable $(\mathrm{Pr})$. Por consiguiente, todos los constructos cumplieron el criterio de Fornell-Larcker.

La segunda prueba fue el radio heterotrait-monotrait (HTMT), en la que los valores deben estar por debajo del umbral de 0.90 (Henseler, Ringle y Sarstedt, 2015). En los resultados (tabla 4) se resaltan los valores que superan el valor establecido, sin embargo pertenecen a construcciones de segundo orden como las creencias de autoeficacia $(\mathrm{C})$ con sus dimensiones autoeficacia general $(\mathrm{Cg})$ y autoeficacia académica $(\mathrm{Ca})$. De igual manera la calidad del servicio de biblioteca (B) con sus tres dimensiones: afectiva (Ba), control de la información (Bi) y espacial (Be). Por lo tanto, todos los constructos cumplen con el radio heterotrait-monotrait. Finalmente se evaluaron las cargas cruzadas, en donde las cargas de los ítems del mismo constructo deben ser mayores que el resto (Lowry y Gaskin, 2014). Todos los valores cumplieron el criterio; por lo tanto, el modelo presentó validez discriminante.

Tabla 1. Operación de los constructos

\begin{tabular}{|c|c|c|c|}
\hline Constructo & Dimensionalidad & Items & Escala de medición \\
\hline \multirow{9}{*}{$\begin{array}{l}\text { Calidad del servicio de } \\
\text { la biblioteca (Green y } \\
\text { Kyrillidou, 2012) }\end{array}$} & \multirow{9}{*}{ Dimensión afectiva } & B1. La confianza que inspira el personal de la biblioteca & \multirow{9}{*}{$\begin{array}{l}\text { Nivel mínimo esperado, } \\
\text { nivel deseado y nivel } \\
\text { esperado del servicio. } \\
\text { Evaluados en una escala } \\
\text { del } 1 \text { al } 9\end{array}$} \\
\hline & & $\begin{array}{l}\text { B2. La disposición del personal de la biblioteca para responder las } \\
\text { preguntas de los usuarios }\end{array}$ & \\
\hline & & $\begin{array}{l}\text { B3. La voluntad que tiene el personal de la biblioteca para ayudar a los } \\
\text { usuarios }\end{array}$ & \\
\hline & & $\begin{array}{l}\text { B4. La fiabilidad que muestra el personal de la biblioteca en el } \\
\text { tratamiento de los problemas del servicio manifestados por los } \\
\text { usuarios }\end{array}$ & \\
\hline & & B5. La atención personalizada que ofrece el personal de la biblioteca & \\
\hline & & $\begin{array}{l}\text { B6. El conocimiento que tiene el personal de la biblioteca para } \\
\text { responder las preguntas de los usuarios }\end{array}$ & \\
\hline & & B7. La amabilidad constante del personal de la biblioteca & \\
\hline & & $\begin{array}{l}\text { B8. Los buenos modales con los que el personal de la biblioteca trata } \\
\text { a los usuarios }\end{array}$ & \\
\hline & & $\begin{array}{l}\text { B9. La comprensión del personal de la biblioteca hacia las necesidades } \\
\text { de los usuarios }\end{array}$ & \\
\hline
\end{tabular}




\begin{tabular}{|c|c|c|c|}
\hline Constructo & Dimensionalidad & Items & Escala de medición \\
\hline \multirow{13}{*}{$\begin{array}{l}\text { Calidad del servicio de } \\
\text { la biblioteca (Green y } \\
\text { Kyrillidou, 2012) }\end{array}$} & \multirow{8}{*}{$\begin{array}{l}\text { Dimensión control de la } \\
\text { información }\end{array}$} & $\begin{array}{l}\text { B10. Las colecciones de la biblioteca como son revistas impresas y /o } \\
\text { electrónicas que necesito para mi trabajo académico }\end{array}$ & \multirow{13}{*}{$\begin{array}{l}\text { Nivel mínimo esperado, } \\
\text { nivel deseado y nivel } \\
\text { esperado del servicio. } \\
\text { Evaluados en una escala } \\
\text { del } 1 \text { al } 9\end{array}$} \\
\hline & & $\begin{array}{l}\text { B11. Los materiales impresos con los que cuenta la biblioteca que } \\
\text { necesito para mi trabajo académico }\end{array}$ & \\
\hline & & $\begin{array}{l}\text { B12. Los recursos de información electrónica ofrecidos por la biblioteca } \\
\text { que necesito para mi trabajo }\end{array}$ & \\
\hline & & $\begin{array}{l}\text { B13. Las herramientas de la biblioteca de fácil uso y acceso que } \\
\text { permiten encontrar información por mí mismo(a) }\end{array}$ & \\
\hline & & $\begin{array}{l}\text { B14. La página web de la biblioteca me permite encontrar información } \\
\text { por mí mismo(a) }\end{array}$ & \\
\hline & & $\begin{array}{l}\text { B15. Los equipos modernos de la biblioteca que me permiten acceder } \\
\text { fácilmente a la información que requiero }\end{array}$ & \\
\hline & & $\begin{array}{l}\text { B16. El fácil acceso a la información de la biblioteca para un uso } \\
\text { autónomo }\end{array}$ & \\
\hline & & $\begin{array}{l}\text { B17. La accesibilidad de los recursos electrónicos de la biblioteca desde } \\
\text { casa u oficina }\end{array}$ & \\
\hline & \multirow{5}{*}{ Dimensión espacial } & $\begin{array}{l}\text { B18. Los espacios tranquilos para las actividades individuales con los } \\
\text { que cuenta la biblioteca }\end{array}$ & \\
\hline & & B19. Las instalaciones confortables y acogedoras de la biblioteca & \\
\hline & & B20. El espacio de la biblioteca invita al estudio y al aprendizaje & \\
\hline & & $\begin{array}{l}\text { B21. El espacio comunitario de la biblioteca para aprendizaje y estudio } \\
\text { de grupos }\end{array}$ & \\
\hline & & $\begin{array}{l}\text { B22. La posibilidad de estudiar, aprender o investigar dentro de la } \\
\text { biblioteca }\end{array}$ & \\
\hline \multirow{7}{*}{$\begin{array}{l}\text { Creencias de autoeficacia } \\
\text { (Baessler y Schwarzer, } \\
\text { 1996; Palenzuela 1983) }\end{array}$} & \multirow{7}{*}{ Autoeficacia general } & $\begin{array}{l}\text { C1. Puedo encontrar la forma de obtener lo que quiero aunque alguien } \\
\text { se me oponga }\end{array}$ & \multirow{7}{*}{$\begin{array}{l}\text { Totalmente en } \\
\text { desacuerdo, 2, 3, 4, 5, } \\
\text { totalmente de acuerdo }\end{array}$} \\
\hline & & C2. Puedo resolver problemas difíciles si me esfuerzo lo suficiente & \\
\hline & & $\begin{array}{l}\text { C3. Me es fácil persistir en lo que me he propuesto hasta llegar a } \\
\text { alcanzar mis metas }\end{array}$ & \\
\hline & & $\begin{array}{l}\text { C4. Tengo confianza en que podría manejar eficazmente } \\
\text { acontecimientos inesperados }\end{array}$ & \\
\hline & & $\begin{array}{l}\text { C5. Gracias a mis cualidades y recursos puedo superar situaciones } \\
\text { imprevistas }\end{array}$ & \\
\hline & & $\begin{array}{l}\text { C6. Cuando me encuentro en dificultades puedo permanecer } \\
\text { tranquilo porque cuento con las habilidades necesarias para manejar } \\
\text { situaciones difíciles }\end{array}$ & \\
\hline & & C7. Venga lo que venga, por lo general soy capaz de manejarlo & \\
\hline
\end{tabular}




\begin{tabular}{|c|c|c|c|}
\hline Constructo & Dimensionalidad & Items & Escala de medición \\
\hline \multirow{13}{*}{$\begin{array}{l}\text { Creencias de autoeficacia } \\
\text { (Baessler y Schwarzer, } \\
\text { 1996; Palenzuela 1983) }\end{array}$} & \multirow{3}{*}{ Autoeficacia general } & $\begin{array}{l}\text { C8. Puedo resolver la mayoría de los problemas si me esfuerzo lo } \\
\text { necesario }\end{array}$ & \multirow{13}{*}{$\begin{array}{l}\text { Totalmente en } \\
\text { desacuerdo, 2, 3, 4, 5, } \\
\text { totalmente de acuerd }\end{array}$} \\
\hline & & $\begin{array}{l}\text { C9. Si me encuentro en una situación difícil, generalmente se me } \\
\text { ocurre qué debo hacer }\end{array}$ & \\
\hline & & $\begin{array}{l}\text { C10. Al tener que hacer frente a un problema, generalmente se me } \\
\text { ocurren varias alternativas de cómo resolverlo }\end{array}$ & \\
\hline & \multirow{10}{*}{ Autoeficacia académica } & $\begin{array}{l}\text { C11. Me considero lo suficientemente capacitado para enfrentarme } \\
\text { con éxito a cualquier tarea académica }\end{array}$ & \\
\hline & & $\begin{array}{l}\text { C12. Pienso que tengo bastante capacidad para comprender bien y } \\
\text { con rapidez una materia }\end{array}$ & \\
\hline & & $\begin{array}{l}\text { C13. Me siento con confianza para abordar situaciones que ponen a } \\
\text { prueba mi capacidad académica }\end{array}$ & \\
\hline & & C14. Tengo la convicción de que puedo hacer exámenes excelentes & \\
\hline & & $\begin{array}{l}\text { C15. No me importa que los profesores sean exigentes y duros, pues } \\
\text { confío mucho en mi propia capacidad académica** }\end{array}$ & \\
\hline & & $\begin{array}{l}\text { C16. Creo que soy una persona bastante capacitada y competente en } \\
\text { mi vida académica }\end{array}$ & \\
\hline & & $\begin{array}{l}\text { C17. Si me lo propongo, creo que tengo la suficiente capacidad para } \\
\text { obtener un buen expediente académico }\end{array}$ & \\
\hline & & $\begin{array}{l}\text { C18. Pienso que puedo pasar los cursos con bastante facilidad, e } \\
\text { incluso, sacar buenas notas }\end{array}$ & \\
\hline & & $\begin{array}{l}\text { C19. Soy de esas personas que no necesito estudiar para aprobar una } \\
\text { asignatura o pasar un curso completo }\end{array}$ & \\
\hline & & $\begin{array}{l}\text { C20. Creo que estoy preparado y bastante capacitado para conseguir } \\
\text { muchos éxitos académicos }\end{array}$ & \\
\hline \multirow{7}{*}{$\begin{array}{l}\text { Participación del } \\
\text { estudiante en la } \\
\text { biblioteca (Yi y Gong, } \\
2013 \text { ) }\end{array}$} & \multirow{3}{*}{$\begin{array}{l}\text { Búsqueda de } \\
\text { información }\end{array}$} & P1. He pedido a otros información sobre lo que la biblioteca ofrece & \multirow{7}{*}{$\begin{array}{l}\text { Totalmente en } \\
\text { desacuerdo, 2, 3, 4, 5, 6, } \\
\text { totalmente de acuerdo }\end{array}$} \\
\hline & & P2. He buscado información sobre dónde está localizada la biblioteca & \\
\hline & & $\begin{array}{l}\text { P3. He prestado atención sobre cómo se comportan los demás para } \\
\text { utilizar bien la biblioteca }\end{array}$ & \\
\hline & \multirow{3}{*}{ Compartir información } & $\begin{array}{l}\text { P4. He explicado claramente lo que quería que el personal de la } \\
\text { biblioteca hiciera }\end{array}$ & \\
\hline & & P5. He dado la información adecuada al personal de la biblioteca & \\
\hline & & $\begin{array}{l}\text { P6. He proporcionado la información necesaria para que el personal de } \\
\text { la biblioteca pueda realizar su tarea }\end{array}$ & \\
\hline & $\begin{array}{l}\text { Comportamiento } \\
\text { responsable }\end{array}$ & $\begin{array}{l}\text { P7 He respondido al personal de la biblioteca todas las preguntas } \\
\text { relacionadas con el servicio }\end{array}$ & \\
\hline
\end{tabular}




\begin{tabular}{|c|c|c|c|}
\hline Constructo & Dimensionalidad & Items & Escala de medición \\
\hline \multirow{9}{*}{$\begin{array}{l}\text { Participación del } \\
\text { estudiante en la } \\
\text { biblioteca (Yi y Gong, } \\
\text { 2013) }\end{array}$} & \multirow{4}{*}{$\begin{array}{l}\text { Comportamiento } \\
\text { responsable }\end{array}$} & P8. He realizado todas las tareas que se requieren & \multirow{9}{*}{$\begin{array}{l}\text { Totalmente en } \\
\text { desacuerdo, 2, 3, 4, 5, 6, } \\
\text { totalmente de acuerdo }\end{array}$} \\
\hline & & $\begin{array}{l}\text { P9. He completado adecuadamente todos los comportamientos } \\
\text { esperados }\end{array}$ & \\
\hline & & P10. He seguido las directivas u órdenes del personal de la biblioteca & \\
\hline & & P11. He cumplido mis responsabilidades con la biblioteca & \\
\hline & \multirow{5}{*}{ Interacción personal } & P12. He sido amigable con el personal de la biblioteca & \\
\hline & & P13. He sido amable con el personal de la biblioteca & \\
\hline & & P14. He sido educado con el personal de la biblioteca & \\
\hline & & P15. He sido cortés con el personal de la biblioteca & \\
\hline & & P16. No he actuado groseramente con el personal de la biblioteca & \\
\hline \multirow{11}{*}{$\begin{array}{l}\text { Estrategias de aprendizaje } \\
\text { (Román y Gallego, } \\
\text { 1994) }\end{array}$} & \multirow{11}{*}{$\begin{array}{l}\text { Estrategias de apoyo al } \\
\text { aprendizaje }\end{array}$} & $\begin{array}{l}\text { E1. Estudio para ampliar mis conocimientos, para saber más, para ser } \\
\text { más experto(a) }\end{array}$ & \multirow{11}{*}{$\begin{array}{l}\text { Totalmente en } \\
\text { desacuerdo, } 2,3,4 \text {, } \\
\text { totalmente de acuerdo }\end{array}$} \\
\hline & & $\begin{array}{l}\text { E2. Me esfuerzo en el estudio para sentirme orgulloso (a) de mí } \\
\text { mismo(a) }\end{array}$ & \\
\hline & & $\begin{array}{l}\text { E3. Me dirijo a mí mismo(a) palabras de ánimo para estimularme y } \\
\text { mantenerme en las tareas de estudio }\end{array}$ & \\
\hline & & $\begin{array}{l}\text { E4. Me digo a mí mismo(a) que puedo superar mi nivel de } \\
\text { rendimiento actual (expectativas) en las distintas asignaturas }\end{array}$ & \\
\hline & & $\begin{array}{l}\text { E5. Pongo en juego recursos personales para controlar mis estados de } \\
\text { ansiedad cuando me impiden concentrarme en el estudio }\end{array}$ & \\
\hline & & $\begin{array}{l}\text { E6. Procuro que en el lugar de estudio no haya nada que pueda } \\
\text { distraerme, como personas, ruidos, desorden, falta de luz, ventilación, } \\
\text { etcétera. }\end{array}$ & \\
\hline & & $\begin{array}{l}\text { E7. Cuando tengo conflictos familiares procuro resolverlos antes, si } \\
\text { puedo, para concentrarme mejor en el estudio }\end{array}$ & \\
\hline & & $\begin{array}{l}\text { E8. En el trabajo, me estimula intercambiar opiniones con mis } \\
\text { compañeros, amigos o familiares sobre temas que estoy estudiando }\end{array}$ & \\
\hline & & $\begin{array}{l}\text { E9. Evito o resuelvo, mediante el diálogo, los conflictos que surgen en la } \\
\text { relación personal con mis compañeros, profesores o familiares }\end{array}$ & \\
\hline & & $\begin{array}{l}\text { E10. Acudo a los amigos, profesores o familiares cuando tengo } \\
\text { dudas o puntos oscuros en los temas de estudio o para intercambiar } \\
\text { información }\end{array}$ & \\
\hline & & $\begin{array}{l}\text { E11. Me satisface que mis compañeros, profesores y familiares valoren } \\
\text { positivamente mi trabajo }\end{array}$ & \\
\hline
\end{tabular}




\begin{tabular}{|c|c|c|c|}
\hline Constructo & Dimensionalidad & Items & Escala de medición \\
\hline \multirow{4}{*}{$\begin{array}{l}\text { Estrategias de aprendizaje } \\
\text { (Román y Gallego, } \\
\text { 1994) }\end{array}$} & \multirow{3}{*}{$\begin{array}{l}\text { Estrategias de apoyo al } \\
\text { aprendizaje }\end{array}$} & $\begin{array}{l}\text { E12. Animo y ayudo a mis compañeros para que obtengan el mayor } \\
\text { éxito posible en las tareas escolares }\end{array}$ & \multirow{4}{*}{$\begin{array}{l}\text { Totalmente en } \\
\text { desacuerdo, 2, 3, 4, } \\
\text { totalmente de acuerdo }\end{array}$} \\
\hline & & $\begin{array}{l}\text { E13. Antes de iniciar el estudio, distribuyo el tiempo de que dispongo } \\
\text { entre todos los temas que tengo que aprender }\end{array}$ & \\
\hline & & $\begin{array}{l}\text { E14. Cuando se acercan los exámenes establezco un plan de trabajo } \\
\text { distribuyendo el tiempo dedicado a cada tema }\end{array}$ & \\
\hline & Hábitos de estudio & $\begin{array}{l}\text { E15. Cuando estoy estudiando una lección, para facilitar la } \\
\text { comprensión, descanso y después la repaso para aprenderla mejor }\end{array}$ & \\
\hline \multirow{12}{*}{$\begin{array}{l}\text { Procesos de } \\
\text { autorregulación del } \\
\text { aprendizaje (Rosário et } \\
\text { al., 2006) }\end{array}$} & & $\begin{array}{l}\text { A1. Hago un plan antes de comenzar a hacer un trabajo escrito. Pienso } \\
\text { lo que voy a hacer y lo que necesito para conseguirlo }\end{array}$ & \multirow{12}{*}{ Nunca, 2, 3, 4, siempre } \\
\hline & & $\begin{array}{l}\text { A2. Después de terminar un examen parcial / final, lo reviso } \\
\text { mentalmente para saber dónde tuve los aciertos y errores y, hacerme } \\
\text { una idea de la nota que voy a tener }\end{array}$ & \\
\hline & & $\begin{array}{l}\text { A3. Cuando estudio, intento comprender las materias, tomar apuntes, } \\
\text { hacer resúmenes, resolver ejercicios, hacer preguntas sobre los } \\
\text { contenidos }\end{array}$ & \\
\hline & & $\begin{array}{l}\text { A4. Cuando recibo una nota, suelo pensar en cosas concretas que } \\
\text { tengo que hacer para mejorar mi rendimiento / nota media }\end{array}$ & \\
\hline & & $\begin{array}{l}\text { A5. Estoy seguro de que soy capaz de comprender lo que me van a } \\
\text { enseñar y por eso creo que voy a tener buenas notas }\end{array}$ & \\
\hline & & $\begin{array}{l}\text { A6. Cumplo mis horarios de estudio, e introduzco pequeños cambios } \\
\text { siempre que es necesario }\end{array}$ & \\
\hline & & $\begin{array}{l}\text { A7. Guardo y analizo las correcciones de los trabajos escritos / } \\
\text { parciales..., para ver dónde me equivoqué y saber qué tengo que } \\
\text { cambiar para mejorar }\end{array}$ & \\
\hline & & $\begin{array}{l}\text { A8. Mientras estoy en clase o estudiando, si me distraigo o pierdo el } \\
\text { hilo, suelo hacer algo para volver a la tarea y alcanzar mis objetivos }\end{array}$ & \\
\hline & & A9. Establezco objetivos académicos concretos para cada asignatura & \\
\hline & & A10. Busco un sitio tranquilo donde pueda estar concentrado para estudiar & \\
\hline & & $\begin{array}{l}\text { A11. Comparo las notas que saco con los objetivos que me había } \\
\text { marcado para esa asignatura. }\end{array}$ & \\
\hline & & $\begin{array}{l}\text { A12. Antes de comenzar a estudiar, compruebo si tengo todo lo que } \\
\text { necesito: diccionarios, libros, lápices, cuadernos, fotocopias... para no } \\
\text { estar siempre interrumpiendo mi estudio }\end{array}$ & \\
\hline $\begin{array}{l}\text { Rendimiento } \\
\text { académico }\end{array}$ & $\begin{array}{l}\text { Promedio académico } \\
\text { acumulado }\end{array}$ & \multicolumn{2}{|l|}{ R1. ¿Cuál es tu promedio en la licenciatura al momento? } \\
\hline
\end{tabular}


Tabla 2. Resultados del modelo de medida

\begin{tabular}{|c|c|c|c|c|c|c|}
\hline Constructo & Dimensión & Items & $\operatorname{Cargas}(\lambda)$ & $\begin{array}{c}\text { Alfa de } \\
\text { Cronbach (a) }\end{array}$ & $\begin{array}{c}\text { Fiabilidad } \\
\text { compuesta } \\
\text { (рc) }\end{array}$ & AVE \\
\hline \multirow{14}{*}{$\begin{array}{l}\text { Calidad del servicio } \\
\text { bibliotecario (B) }\end{array}$} & \multirow{6}{*}{ Dimensión afectiva $(\mathrm{Ba})$} & B2 & 0.820 & \multirow{6}{*}{0.900} & \multirow{6}{*}{0.923} & \multirow{6}{*}{0.668} \\
\hline & & B3 & 0.847 & & & \\
\hline & & B4 & 0.831 & & & \\
\hline & & B5 & 0.827 & & & \\
\hline & & B6 & 0.798 & & & \\
\hline & & B9 & 0.778 & & & \\
\hline & \multirow{4}{*}{$\begin{array}{l}\text { Control de la } \\
\text { información (Bi) }\end{array}$} & B13 & 0.868 & \multirow{4}{*}{0.865} & \multirow{4}{*}{0.908} & \multirow{4}{*}{0.712} \\
\hline & & B14 & 0.828 & & & \\
\hline & & B15 & 0.850 & & & \\
\hline & & B16 & 0.829 & & & \\
\hline & \multirow{4}{*}{ Dimensión espacial (Be) } & B19 & 0.828 & \multirow{4}{*}{0.882} & \multirow{4}{*}{0.919} & \multirow{4}{*}{0.739} \\
\hline & & B20 & 0.876 & & & \\
\hline & & B21 & 0.871 & & & \\
\hline & & B22 & 0.862 & & & \\
\hline \multirow{9}{*}{$\begin{array}{l}\text { Creencias de } \\
\text { autoeficacia (C) }\end{array}$} & \multirow{9}{*}{$\begin{array}{l}\text { Autoeficacia general } \\
\text { (Cg) }\end{array}$} & C2 & 0.791 & \multirow{9}{*}{0.926} & \multirow{9}{*}{0.938} & \multirow{9}{*}{0.629} \\
\hline & & C3 & 0.810 & & & \\
\hline & & C4 & 0.810 & & & \\
\hline & & C5 & 0.829 & & & \\
\hline & & C6 & 0.708 & & & \\
\hline & & C7 & 0.814 & & & \\
\hline & & $\mathrm{C} 8$ & 0.799 & & & \\
\hline & & C9 & 0.813 & & & \\
\hline & & $\mathrm{C} 10$ & 0.757 & & & \\
\hline
\end{tabular}




\begin{tabular}{|c|c|c|c|c|c|c|}
\hline Constructo & Dimensión & Items & $\operatorname{Cargas}(\lambda)$ & $\begin{array}{c}\text { Alfa de } \\
\text { Cronbach (a) }\end{array}$ & $\begin{array}{c}\text { Fiabilidad } \\
\text { compuesta } \\
(\rho c)\end{array}$ & AVE \\
\hline \multirow{9}{*}{$\begin{array}{l}\text { Creencias de } \\
\text { autoeficacia (C) }\end{array}$} & \multirow{9}{*}{$\begin{array}{l}\text { Autoeficacia académica } \\
\text { (Ca) }\end{array}$} & C11 & 0.825 & \multirow{9}{*}{0.919} & \multirow{9}{*}{0.933} & \multirow{9}{*}{0.609} \\
\hline & & $\mathrm{C} 12$ & 0.812 & & & \\
\hline & & C13 & 0.818 & & & \\
\hline & & C 14 & 0.753 & & & \\
\hline & & C15 & 0.722 & & & \\
\hline & & C16 & 0.814 & & & \\
\hline & & $\mathrm{C} 17$ & 0.793 & & & \\
\hline & & C18 & 0.724 & & & \\
\hline & & $\mathrm{C} 20$ & 0.752 & & & \\
\hline \multirow{13}{*}{$\begin{array}{l}\text { Participación del } \\
\text { estudiante en la } \\
\text { biblioteca }(P)\end{array}$} & \multirow{3}{*}{$\begin{array}{l}\text { Compartir información } \\
\text { (Ps) }\end{array}$} & P4 & 0.882 & \multirow{3}{*}{0.906} & \multirow{3}{*}{0.941} & \multirow{3}{*}{0.842} \\
\hline & & P5 & 0.950 & & & \\
\hline & & P6 & 0.920 & & & \\
\hline & \multirow{5}{*}{$\begin{array}{l}\text { Comportamiento } \\
\text { responsable }(\mathrm{Pr})\end{array}$} & P7 & 0.775 & \multirow{5}{*}{0.878} & \multirow{5}{*}{0.911} & \multirow{5}{*}{0.673} \\
\hline & & P8 & 0.823 & & & \\
\hline & & P9 & 0.856 & & & \\
\hline & & P10 & 0.842 & & & \\
\hline & & P11 & 0.804 & & & \\
\hline & \multirow{5}{*}{$\begin{array}{l}\text { Interacción personal } \\
(\mathrm{Pp})\end{array}$} & P12 & 0.895 & \multirow{5}{*}{0.924} & \multirow{5}{*}{0.944} & \multirow{5}{*}{0.773} \\
\hline & & P13 & 0.927 & & & \\
\hline & & P14 & 0.914 & & & \\
\hline & & P15 & 0.923 & & & \\
\hline & & P16 & 0.718 & & & \\
\hline
\end{tabular}




\begin{tabular}{|c|c|c|c|c|c|c|}
\hline Constructo & Dimensión & Items & $\operatorname{Cargas}(\lambda)$ & $\begin{array}{c}\text { Alfa de } \\
\text { Cronbach (a) }\end{array}$ & $\begin{array}{c}\text { Fiabilidad } \\
\text { compuesta } \\
(\rho c)\end{array}$ & AVE \\
\hline \multirow{7}{*}{$\begin{array}{l}\text { Estrategias de } \\
\text { aprendizaje (E) }\end{array}$} & & E10 & 0.737 & \multirow{7}{*}{0.860} & \multirow{7}{*}{0.893} & \multirow{7}{*}{0.543} \\
\hline & & E11 & 0.763 & & & \\
\hline & & E12 & 0.724 & & & \\
\hline & & E2 & 0.732 & & & \\
\hline & & E3 & 0.712 & & & \\
\hline & & E4 & 0.733 & & & \\
\hline & & E9 & 0.756 & & & \\
\hline \multirow{5}{*}{$\begin{array}{l}\text { Procesos de } \\
\text { autorregulación del } \\
\text { aprendizaje (A) }\end{array}$} & & A3 & 0.735 & \multirow{5}{*}{0.791} & \multirow{5}{*}{0.857} & \multirow{5}{*}{0.545} \\
\hline & & A4 & 0.737 & & & \\
\hline & & A6 & 0.732 & & & \\
\hline & & A7 & 0.786 & & & \\
\hline & & A9 & 0.700 & & & \\
\hline $\begin{array}{l}\text { Rendimiento } \\
\text { académico (R) }\end{array}$ & $\begin{array}{l}\text { Promedio académico } \\
\text { acumulado }\end{array}$ & R & 1.000 & 1.000 & 1.000 & 1.000 \\
\hline
\end{tabular}


Tabla 3. Criterio de Fornell-Larcker

\begin{tabular}{|l|c|c|c|c|c|c|c|c|c|c|c|c|c|c|}
\hline & Ca & Ba & $\boldsymbol{C}$ & $\boldsymbol{A}$ & $\boldsymbol{B}$ & $\boldsymbol{P s}$ & $\boldsymbol{P r}$ & $\boldsymbol{B i}$ & $\boldsymbol{B e}$ & $\boldsymbol{E}$ & $\boldsymbol{C g}$ & $\boldsymbol{P p}$ & $\boldsymbol{P}$ & $\boldsymbol{R}$ \\
\hline $\mathrm{Ca}$ & 0.780 & & & & & & & & & & & & \\
\hline $\mathrm{Ba}$ & 0.131 & 0.817 & & & & & & & & & & & \\
\hline C & 0.944 & 0.142 & 0.744 & & & & & & & & & & & \\
\hline $\mathrm{A}$ & 0.411 & 0.106 & 0.431 & 0.738 & & & & & & & & & & \\
\hline $\mathrm{B}$ & 0.149 & 0.867 & 0.156 & 0.123 & 0.708 & & & & & & & & & \\
\hline $\mathrm{PS}$ & 0.251 & 0.140 & 0.256 & 0.187 & 0.180 & 0.918 & & & & & & & & \\
\hline $\mathrm{Pr}$ & 0.321 & 0.181 & 0.338 & 0.258 & 0.236 & 0.687 & 0.820 & & & & & & & \\
\hline $\mathrm{Bi}$ & 0.125 & 0.555 & 0.122 & 0.127 & 0.830 & 0.165 & 0.215 & 0.844 & & & & & & \\
\hline $\mathrm{Be}$ & 0.120 & 0.550 & 0.129 & 0.081 & 0.833 & 0.157 & 0.209 & 0.612 & 0.859 & & & & & \\
\hline $\mathrm{E}$ & 0.420 & 0.183 & 0.446 & 0.351 & 0.210 & 0.284 & 0.373 & 0.167 & 0.180 & 0.737 & & & & \\
\hline $\mathrm{Cg}$ & 0.788 & 0.137 & 0.948 & 0.404 & 0.147 & 0.233 & 0.317 & 0.106 & 0.124 & 0.422 & 0.793 & & & \\
\hline $\mathrm{Cp}$ & 0.385 & 0.151 & 0.424 & 0.231 & 0.159 & 0.346 & 0.618 & 0.124 & 0.124 & 0.456 & 0.415 & 0.879 & & \\
\hline $\mathrm{P}$ & 0.389 & 0.189 & 0.416 & 0.273 & 0.229 & 0.750 & 0.923 & 0.199 & 0.194 & 0.453 & 0.396 & 0.827 & 0.729 & \\
\hline $\mathrm{R}$ & 0.165 & 0.011 & 0.132 & 0.191 & 0.002 & 0.037 & 0.065 & -0.007 & -0.003 & 0.137 & 0.085 & 0.046 & 0.061 & 1.000 \\
\hline
\end{tabular}

Tabla 4. Radio heterotrait-monotrait

\begin{tabular}{|c|c|c|c|c|c|c|c|c|c|c|c|c|c|c|}
\hline & $\mathrm{Ca}$ & $B a$ & $C$ & $A$ & B & Ps & $\mathrm{Pr}$ & $B i$ & $B e$ & $E$ & $\mathrm{Cg}$ & $P p$ & $P$ & $R$ \\
\hline \multicolumn{15}{|l|}{$\mathrm{Ca}$} \\
\hline $\mathrm{Ba}$ & 0.143 & & & & & & & & & & & & & \\
\hline C & 1.009 & 0.152 & & & & & & & & & & & & \\
\hline A & 0.484 & 0.125 & 0.497 & & & & & & & & & & & \\
\hline B & 0.161 & 0.958 & 0.166 & 0.146 & & & & & & & & & & \\
\hline Ps & 0.274 & 0.156 & 0.274 & 0.223 & 0.197 & & & & & & & & & \\
\hline $\operatorname{Pr}$ & 0.357 & 0.204 & 0.368 & 0.308 & 0.262 & 0.772 & & & & & & & & \\
\hline $\mathrm{Bi}$ & 0.140 & 0.626 & 0.135 & 0.155 & 0.924 & 0.185 & 0.246 & & & & & & & \\
\hline $\mathrm{Be}$ & 0.132 & 0.616 & 0.140 & 0.100 & 0.916 & 0.176 & 0.238 & 0.699 & & & & & & \\
\hline$E$ & 0.468 & 0.208 & 0.490 & 0.420 & 0.236 & 0.320 & 0.426 & 0.193 & 0.208 & & & & & \\
\hline $\mathrm{Cg}$ & 0.850 & 0.149 & 1.007 & 0.471 & 0.159 & 0.252 & 0.351 & 0.119 & 0.138 & 0.471 & & & & \\
\hline$C p$ & 0.416 & 0.164 & 0.450 & 0.268 & 0.170 & 0.374 & 0.677 & 0.135 & 0.134 & 0.510 & 0.446 & & & \\
\hline P & 0.423 & 0.207 & 0.443 & 0.318 & 0.246 & 0.818 & 1.017 & 0.220 & 0.214 & 0.508 & 0.428 & 0.895 & & \\
\hline $\mathrm{R}$ & 0.174 & 0.021 & 0.135 & 0.213 & 0.020 & 0.039 & 0.070 & 0.007 & 0.021 & 0.148 & 0.087 & 0.050 & 0.064 & \\
\hline
\end{tabular}




\section{Resultados del modelo estructural}

Se utilizó la técnica bootstrapping con 500 submuestras para determinar la significancia de los coeficientes path ( $\beta$ ). Los resultados que se muestran en la tabla 5 muestran que todos los efectos entre los constructos fueron directos, positivos y significativos (valor $\mathrm{t}>1.96$ ), por lo que todas las relaciones fueron validadas. Además, los coeficientes path mostraron un efecto bajo en cuatro relaciones: calidad del servicio de biblioteca con creencias de autoeficacia, participación del alumno en la biblioteca y estrategias de aprendizaje con procesos de autorregulación del aprendizaje, estrategias de aprendizaje con procesos de autorregulación del aprendizaje y con rendimiento académico. Además, los resultados indican que el modelo explica el 3.8\% de la varianza del rendimiento académico (figura 4.1).

Esta investigación encontró que la calidad del servicio bibliotecario, considerada en función de sus dimensiones afectiva, espacial y control de la información, incide de manera significativa en las creencias de los usuarios respecto a su capacidad para identificar y gestionar los recursos e información necesarios para involucrarse en sus actividades académicas orientadas a un mejor aprendizaje, esto es, sus creencias de autoeficacia general y académica. La importancia de este hallazgo radica en que estas creencias estimulan un mayor uso de los servicios bibliotecarios y, a su vez, contribuyen a una mayor diversificación de las estrategias de aprendizaje de los estudiantes.

Las estrategias de aprendizaje, por su parte, tienen una mayor influencia en la autorregulación del aprendizaje, lo que implica que los estudiantes tendrán mayores oportunidades de planear, supervisar y evaluar la efectividad de sus estrategias, que contribuyan a su óptimo desempeño académico. Finalmente, se corroboró que la autorregulación del aprendizaje contribuye de manera significativa en el rendimiento académico de los estudiantes.

Tabla 5. Resultados del modelo estructural

\begin{tabular}{|c|c|c|}
\hline Efectos & $\beta$ & Valor $t$ \\
\hline Calidad del servicio bibliotecario $\rightarrow$ Dimensión afectiva & 0.868 & $58.064^{* * *}$ \\
\hline Calidad del servicio bibliotecario $\rightarrow$ Control de la información & 0.832 & $52.527^{* * *}$ \\
\hline Calidad del servicio bibliotecario $\rightarrow$ Dimensión espacial & 0.835 & $54.050^{* * * *}$ \\
\hline Calidad del servicio bibliotecario $\rightarrow$ Creencias de autoeficacia & 0.161 & $3.634^{* * *}$ \\
\hline Creencias de autoeficacia $\rightarrow$ Autoeficacia general & 0.948 & $155.416^{* * *}$ \\
\hline Creencias de autoeficacia $\rightarrow$ Autoeficacia académica & 0.944 & $137.073^{* * *}$ \\
\hline Creencias de autoeficacia $\rightarrow$ Participación del estudiante en la biblioteca & 0.417 & $9.521^{* * *}$ \\
\hline Participación del estudiante en la biblioteca $\rightarrow$ Compartir información & 0.750 & $38.411^{* * *}$ \\
\hline Participación del estudiante en la biblioteca $\rightarrow$ Comportamiento responsable & 0.923 & $159.151^{* * *}$ \\
\hline Participación del estudiante en la biblioteca $\rightarrow$ Interacción personal & 0.826 & $41.032^{* * *}$ \\
\hline Creencias de autoeficacia $\rightarrow$ Estrategias de aprendizaje & 0.449 & $10.640^{* * *}$ \\
\hline Participación del estudiante en la biblioteca $\rightarrow$ Procesos de autorregulación del aprendizaje & 0.142 & $3.458^{* *}$ \\
\hline Estrategias de aprendizaje $\rightarrow$ Procesos de autorregulación del aprendizaje & 0.293 & $6.295^{* * *}$ \\
\hline Procesos de autorregulación del aprendizaje $\rightarrow$ Rendimiento académico & 0.192 & $5.064^{* * *}$ \\
\hline
\end{tabular}

Nota: nivel de significancia para una t de student de dos colas (499). Elaboración propia. ${ }^{*} p<0.05,{ }^{* *} p<0.01,{ }^{* * *} p<0.001$, n.s. $=$ no significativo. $t(0.05 ; 499)=1.967, t(0.01 ; 499)=2.590, t(0.001 ; 499)=3.319$ 


\section{Figura 4. Resultado del modelo de ecuaciones estructurales (SEM), hipótesis, coeficientes path $(\beta)$ y coeficientes de determinación $\left(\mathbf{R}^{2}\right)$}

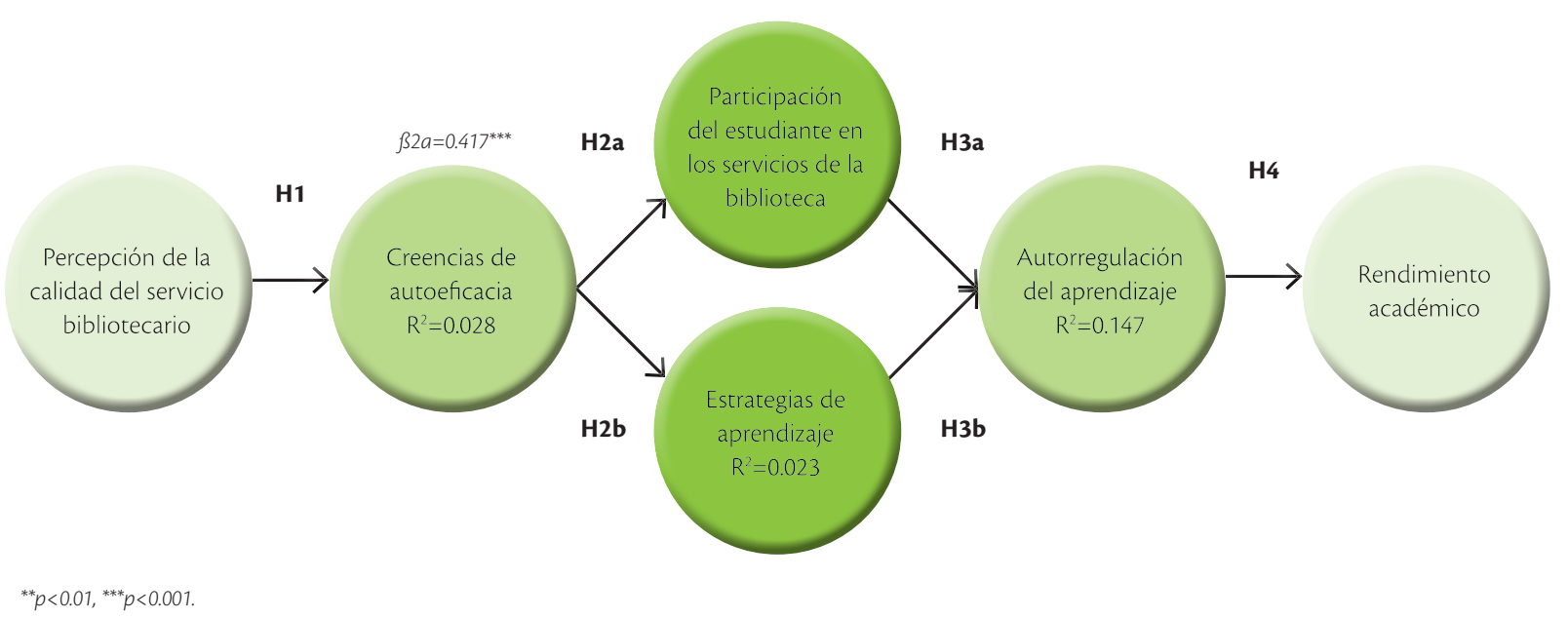

\section{Efectos indirectos}

En el modelo estructural presentado hay cinco efectos indirectos: el efecto de la calidad del servicio bibliotecario sobre la participación del alumno en la biblioteca y las estrategias de aprendizaje; el impacto de las creencias de autoeficacia sobre los procesos de autorregulación del aprendizaje. Además, tanto la participación de los alumnos en la biblioteca como las estrategias de aprendizaje afectan el rendimiento académico y finalmente, el efecto de las estrategias de aprendizaje en el rendimiento académico. Todos los efectos indirectos muestran un impacto bajo, pero positivo y significativo entre constructos (valor $\mathrm{t}>1.96)$.

Estos resultados demuestran la necesidad de que la universidad invierta en la calidad del servicio bibliotecario, ya que no solamente se encontró un efecto directo de ésta sobre las creencias de autoeficacia, sino que indirectamente influye en el resto de los constructos estudiados, por tanto, puede afirmarse que la calidad del servicio de la biblioteca universitaria contribuye a la formación de los estudiantes. Un estudio reciente (Ghaedi, Valizadeh-Haghi, Ahmadi,
Zeraatkar y Baghestani, 2020) encontró que, desafortunadamente, las bibliotecas universitarias han sido consideradas un servicio periférico que, muchas veces, no cubren las expectativas mínimas de sus usuarios, por lo tanto, se requiere prestar más atención a la provisión de este servicio si se desea lograr la mejora académica.

\section{Discusión y conclusiones}

De acuerdo con los resultados de esta investigación se ha evidenciado que varios factores impactan en el rendimiento académico de los estudiantes universitarios; cinco constructos impactan sobre éste. Los resultados confirmaron que la calidad del servicio de biblioteca es un antecedente de las creencias de autoeficacia (H1), efecto que se ha demostrado en estudios previos (Zha et al., 2015). Las creencias de autoeficacia mostraron un efecto directo sobre la participación de los estudiantes en la biblioteca (H2a) y las estrategias de aprendizaje (H2b), lo que también se ha demostrado en varias investigaciones previas (Akilli y Genç, 2017; Alves et al., 2016; Kui y Kun, 2014; Mellat y Lavasani, 2011). 
Los resultados también mostraron que la participación del alumno en la biblioteca (H3a) y las estrategias de aprendizaje (H3b) son predictores de los procesos de autorregulación del aprendizaje; hipótesis apoyadas previamente (Chih-Yuan Sun y Rueda, 2012; Fraile et al., 2017; Mellat y Lavasani, 2011). Finalmente, los procesos de autorregulación del aprendizaje mostraron ser antecedente del rendimiento académico (H4); efecto previamente demostrado en diferentes estudios (Duru et al., 2014; Montroy et al., 2014). Por lo tanto, todas las hipótesis presentadas en esta investigación han sido aceptadas.

Con base en los hallazgos, se concluye que la calidad del servicio de la biblioteca tiene efectos importantes en diferentes aspectos de la vida académica de los estudiantes de licenciatura, que en última instancia afectan su desempeño académico. Estos hallazgos también respaldan los estudios previos, que han demostrado el efecto directo que tiene el uso de la biblioteca en el aprendizaje de los estudiantes (Stemmer y Mahan, 2016; Wong y Webb, 2011). La autoeficacia del alumno, su participación en la biblioteca, las estrategias de aprendizaje y el proceso de autorregulación del aprendizaje son los nuevos elementos con los que el estudio ha contribuido. Estas variables son importantes ya que todas ayudan a los estudiantes a participar activamente en el proceso educativo, perseverar en el logro de objetivos, utilizar diferentes estrategias y obtener calificaciones satisfactorias.

Cabe señalar que en México, como en otros países emergentes, la biblioteca física sigue siendo una fuente importante de recursos. A pesar de que las tecnologías de información se han incorporado, han sido incluidas parcialmente por los servicios bibliotecarios (Tarango y Lau, 2013). Por lo tanto, la biblioteca proporciona acceso a varios servicios para ayudar a los procesos de aprendizaje y enseñanza a través de material impreso y digital, de igual manera también representa un espacio social y multifuncional donde los estudiantes pueden reunirse para realizar diversas tareas (Varela y Baiget, 2012).

Algunas limitaciones deben abordarse cuando se establecen las conclusiones y futuras investigaciones. La primera tiene que ver con la selección de participantes, para obtener resultados representativos de toda la población; se sugiere realizar una investigación con muestreo probabilístico, ya que en esta investigación solamente participaron cuatro universidades. En segundo lugar, dado que los datos recopilados se basan sólo en cuestionarios, faltan datos cualitativos detallados como entrevistas con estudiantes y personal de la biblioteca para conocer los motivos de uso de los diferentes servicios bibliotecarios. Además, las investigaciones futuras deberían tratar de encontrar otros posibles efectos de la calidad del servicio de la biblioteca en la vida académica del alumno, como la motivación y el compromiso del alumno, teniendo en cuenta los efectos moderadores de la disciplina de estudio, el género y los antecedentes del alumno. A la luz de los resultados obtenidos, es necesario que las universidades inviertan recursos en los servicios de la biblioteca con el fin de mejorar la calidad de la educación de los estudiantes. 


\section{Referencias}

Alves, H., J. J. Ferreira y G. I. Fernandes (2016), "Customer's operant resources effects on co-creation activities", fournal of Innovation \&̊ Knowledge, vol. 1, núm 2, pp. 69-80, DOI: 10.1016/j.jik.2016.03.001.

American Library Association (ALA) (2010), American Library Association, ALA, <http://www.ala.org/research/ librarystats/academic $>$ [Consulta: enero de 2017].

Association of College \& Research Libraries (2016), Value of academic libraries statement, EUA, Association of College \& Research Libraries.

Bandura, A. (1993), "Perceived self-efficacy in cognitive development and functioning", Educational Psychologist, vol. 28, pp. 117-148, DOI: 10.1207/s15326985ep 2802_3.

Blanco, A. B. (2010), "Creencias de autoeficacia de estudiantes universitarios: Un estudio empírico sobre la especificidad del constructo", Relieve, vol. 16, núm. 1, pp. 1-28.

Cassidy, S. (2011), "Self-regulated learning in higher education: identifying key component processes", Studies in Higher Education, vol. 36, núm. 8, pp. 989-1000, DOI: $10.1080 / 03075079.2010 .503269$.

Celina Oviedo, H. y A. Campo Arias (2005), "Aproximación al uso del coeficiente alfa de Cronbach", Revista Colombiana de Psiquiatría, vol. 34, núm. 4, pp. 572-580.

Chih-Yuan Sun, J. y R. Rueda (2012), "Situational interest, computer self-efficacy and self-regulation: their impact on student engagement in distance education", British Fournal of Educational Technology, vol. 43, núm. 2, pp. 191-204, DOI: 10.1111/j.1467-8535.2010.01157.x.

Duggal, M. y P. Mehta (2015), "Antecedents to academic performance of college students: An empirical investigation", Paradigm, vol. 19, núm. 2, pp. 197-211, DOI: $10.1177 / 0971890715609992$.

Duru, E., S. Duru y M. Balkis (2014), "Analysis of relationships among burnout, academic achievement and self-regulation", Educational Sciences: Theory Eా Practice, pp. 1274-1284.
Encinas-Orozco, F. C. y J. Cavazos-Arroyo (2017), Co-creación y comportamiento ciudadano del consumidor en el marketing de servicios educativos, México, Pearson Educación de México.

Fiagborlo, J. D. y E. K. Kunu (2016), "An econometric assessment of factors that predict academic performance of tertiary students in Ho, Ghana", Fournal of Economics and Economic Education Research, vol. 17, núm. 3, pp. 47-59.

Fornell, C. y D. Larcker (1981), "Evaluating structural equation models with unobservable variables and measurement error", Journal of Marketing Research, vol. 28, pp. 39-50, DOI: 10.1177/002224378101800104.

Fraile, J., E. Panadero y R. Pardo (2017), “Co-creating rubrics: the effects on self-regulated learning, selfefficacy and performance of establishing assessment criteria with students", Studies in Educational Evaluation, vol.53,pp.69-76, DOI: 10.1016/j.stueduc.2017.03.003.

Ghaedi, R., S. Valizadeh-Haghi, E. Ahmadi, Z. Zeraatkar y A. R. Baghestani (2020), "Gaps between users expectations and their perceptions on service quality of college libraries of Shahid Beheshti University of Medical Sciences: a case study", DESIDOC Journal of Library \& Information Technology, vol. 40, núm. 2, pp. 20-31.

Gandomkar, R., A. Mirzazadeh, M. Jalili, K. Yazdani, L. Fata y J. Sandars (2016), "Self-regulated learning processes of medical students during an academic learning task", Medical Education, pp. 1065-1074, DOI: $10.1111 /$ medu. 12975 .

García, T., E. J. McCann, J. E. Turner y L. Roska (1998), "Modeling the mediating role of volition in the learning process", Contemporary Educational Psychology, vol. 23, pp. 392-418, DOI: 10.1006/ceps.1998.0982.

González, T. M. (1997), La motivación académica. Sus determinantes y pautas de intervención, España, EUNSA.

González Guitián, M. V. y M. Molina Piñeiro (2008), "Las bibliotecas universitarias: breve aproximación a sus nuevos escenarios y retos", Revista Cubana de Información en Ciencias de la Salud, vol. 18, núm. 2. 
Green, D. y M. Kyrillidou (2012), Procedures manual LibQUAL+, Washington, Association of Research Libraries.

Hair, J., R. Bush y D. Ortinau (2010), Investigación de mercados. En un ambiente de información digital, México, $\mathrm{Mc}$ Graw-Hill/ Interamericana Editores.

Henseler, J., C. Ringle y M. Sarstedt (2015), "A new criterion for assessing discriminant validity in variancebased structural equation modeling", fournal of the Academy of Marketing Science, vol. 43, pp. 115-135, DOI: 10.1007/s1 1747-014-0403-8.

Hojo, M. (2012), "Determinants of academic performance in Japan", The Japanese Economy, vol. 39, núm. 3, pp. 3-29, DOI: 10.2753/JES1097-203X390301.

Hyang-Il, K. y C. Kyung-Ae (2017), "Effects of experience abroad and language proficiency on self-efficacy beliefs in language learning", Psychological Reports, vol. 120, núm. 4, pp. 670-694, DOI: 10.1177/0033294117697088.

Kui, X. y H. Kun (2014), "The role of beliefs and motivation in asynchronous online learning in collegelevel classes", Journal of Educational Computing Research, vol. 50, núm. 3, pp. 315-341, DOI: 10.2190/EC.50.3.b .

LibQUAL+ (2016), "Learn About LibQUAL+ Presentation", Charting Library Service Quality, <https:/ / www.libqual.org/about/about_survey/tools $>$ [Consulta: enero de 2017].

Lowry, P. B. y J. Gaskin (2014), "Partial least squares (PLS) structural equation modeling (SEM) for building and testing behavioral causal theory: when to choose it and how to use it", IEEE Transactions on Professional Communication, vol. 57, núm. 2, pp. 123-146, DOI: 10.1109/TPG.2014.2312452.

Luszczynska, A., U. Scholz y R. Schwarzer (2005), "The general self-efficacy scale: multicultural validation studies", The fournal of Psychology, vol. 139, núm. 5, pp. 439-457, DOI: 10.3200/JRLP.139.5.439-457.

Malhotra, N. (2008), Investigación de mercados, México, Pearson Educación.

Mellat, N. y M. G. Lavasani (2011), "The role of epistemological beliefs, motivational constructs and information processing strategies in regulation of learning", Procedia Social and Behavioral Sciences, pp. 17611769, DOI: 10.1016/j.sbspro.2011.10.340.

Montroy, J. J., R. P. Bowles, L. E. Skibbe y T. D. Foster (2014), "Social skills and problem behaviors as mediators of the relationship between behavioral selfregulation and academic achievement", Early Childhood Research Quarterly, vol. 29, pp. 298-309, DOI: 10.1016/j. ecresq.2014.03.002.

Nunnally, J. (1978), Psychometric theory 2, EUA, McGrawHill.

Pajares, F. y T. Urdan (2006), Self-efficacy beliefs of adolescents, EUA, Information Age Publishing.

Rocca, K. A. (2010), "Student participation in the college classroom: an extended multidisciplinary literature review", Communication Education, vol. 59, núm. 2, pp. 185-213, DOI: 10.1080/03634520903505936 .

Roux, R., y E. E. Anzures González (2015), “Estrategias de aprendizaje y su relación con el rendimiento académico en estudiantes de una escuela privada de educación media superior”, Actualidades Investigativas en Educación, vol. 15, núm 1, pp. 1-16.

Stemmer, J. y D. Mahan (2016), "Investigating the relationship of library usage to student outcomes", College E̊ Research Libraries, vol. 77, núm. 3, pp. 359-375.

Tarango, J. y J. Lau (2013), "Educación bibliotecológica, TIC y bibliotecas universitarias: hacia un reenfoque curricular", en Las Tecnologías de la Información y la Comunicación en la educación bibliotecológica y la documentación en Iberoamérica, México, Instituto de Investigaciones Bibliotecológicas y de la Información, UNAM, pp. 109131.

Varela Prado, C. y T. Baiget (2012), "El futuro de las bibliotecas académicas: incertidumbres, oportunidades y retos", Investigación bibliotecnológica, vol. 26, núm. 56, pp. 115-135.

Vargas Hernández, M. M. y E. Montero Rojas (2016), "Factores que determinan el rendimiento académico en Matemáticas en el contexto de una universidad tecnológica: aplicación de un modelo de ecuaciones estructurales", Universitas Psychologica, vol. 15, núm. 4, DOI: 10.11144/Javeriana.upsy 15-4.fdra. 
Visbal-Cadavid, D., A. Mendoza-Mendoza y S. Díaz Santana (2017), "Estrategias de aprendizaje en la educación”, Sophia, vol. 13, núm. 2, pp. 70-81, DOI: 10.18634/sophiaj.13v.2i.461.

Wong, S. y T. D. Webb (2011), "Uncovering meaningful correlation between student academic performance and library material usage", College \& Research Libraries, vol. 72, núm. 4, pp. 361-370.

Yeung, P., A. Passmore y T. Packer (2012), "Examining citizenship participation in young Australian adults: A structural equation analysis", fournal of Touth Studies, vol. 15, núm. 1, pp. 73-98, DOI: 10.1080/13676261.2011.623689.

Young Kim, J. y H. Seok Lee (2013), "Key factors influencing customer satisfaction in Korea's mobile service sector", Fournal of Internet Banking and Commerce, vol. 18, núm. 3, pp. 1-13.

Zha, X., W. Wang, Y. Yan, J. Zhang y D. Zha (2015),
"Understanding information seeking in digital libraries: antecedents and consequences", Aslib fournal of Information Management, vol. 67, núm. 6, pp. 715-734. Zimmer, G. y R. Haumann (2013), Handbook of academic performance: predictors, learning strategies and influences of gender, Nueva York, Nova Science Publishers.

Zimmerman, B. J. y M. Martinez-Pons (1990), "Student differences in self-regulated learning: Relating grade, sex, and giftedness to self-efficacy and strategy use", Fournal of Educational Psychology, vol. 82, pp. 51-59, DOI: 10.1037/0022-0663.82.1.51.

Zimmerman, B. J. y D. H. Schunk (2008), Self-regulated learning and academic achievement: Theoretical perspectives, EUA, Taylor \& Francis.

Zulkosky, K. (2009), "Self-efficacy: a concept analysis", en Nursing fórum, EUA, Blackwell Publishing Inc., pp. 93102.

\section{Cómo citar este artículo:}

Aparicio-Ley, Eloisa, Judith Cavazos-Arroyo y Martha-Leticia Gaeta-González (2021), "La calidad del servicio bibliotecario y el desempeño académico en la universidad: un modelo explicativo", Revista Iberoamericana de Educación Superior (RIES), vol. XII, núm. 35, pp. 185-205, DOI: https://doi.org/10.22201/iisue.20072872e.2021.35.1089 [Consulta: fecha de última consulta]. 